\title{
Ionic Transport Processes
}

In Electrochemistry and Membrane Science

KYÖSTI KONTTURI and LASSE MURTOMÄKI Helsinki University of Technology, Finland

JOSÉ A. MANZANARES

University of Valencia, Spain 


\section{Contents}

\section{Thermodynamics of irreversible processes}

1.1 Fundamental concepts

1.1.1 Space and time scales of observation

1.1.2 Local thermodynamic equations

1.1.3 Electrolyte solutions

1.2 Balance equations

1.2.1 Introduction

1.2.2 General form of the balance equations

1.2.3 Total and component mass-balance equations

1.2.4 Electric charge-balance equation

1.2.5 Linear momentum-balance equation

1.2.6 Energy- and entropy-balance equations in a non-viscous fluid

1.2.7 Energy- and entropy-balance equations in a Newtonian viscous fluid

1.2.8 Electromagnetic energy and linear momentum

Exercises

References

\section{Transport equations}

2.1 Linear phenomenological equations

2.1.1 Introduction

2.1.2 Ionic transport equations

2.1.3 Binary electrolyte solution

2.1.4 Electric conduction

2.1.5 Component transport equations

2.1.6 Ternary electrolyte solutions

2.2 The Fickian approach

2.2.1 Introduction

2.2.2 Fick's law

2.2.3 Diffusion-conduction equations 49

2.3 The Nernst-Planck approximation

2.3.1 Introduction

2.3.2 Nernst-Planck equation

2.3.3 Electrical coupling between the ionic fluxes

2.3.4 Diffusion potential

2.3.5 Integration of the transport equations in multi-ionic solutions 
2.3.6 Binary electrolyte solution $\quad 57$

2.3.7 Ternary electrolyte solutions $\quad 58$

2.3.8 Weak electrolytes 61

2.3.9 Moderately concentrated solutions 63

2.4 The Stefan-Maxwell approach 64

2.4.1 Introduction 64

2.4.2 Diffusion of a neutral component 65

2.4.3 Binary electrolyte solution $\quad 66$

2.4.4 Multi-ionic systems $\quad 69$

Exercises $\quad 70$

$\begin{array}{ll}\text { References } & 76\end{array}$

3 Transport at electrodes $\quad 78$

$\begin{array}{ll}3.1 \text { Faraday's law } & 78\end{array}$

3.2 Electrode processes in stationary state $\quad 80$

3.2.1 Nernst diffusion boundary layer $\quad 80$

3.2.2 Limiting current density 81

3.2.3 Transport equations in multi-ionic solutions $\quad 82$

3.2.4 Trace ions 83

3.2.5 Solutions with only one electroactive ion $\quad 84$

3.2.6 Solutions with several electroactive species $\quad 89$

3.2.7 Transport in spherical geometry $\quad 95$

$\begin{array}{lll}3.3 & \text { Hydrodynamic electrodes } & 98\end{array}$

3.3.1 Rotating-disc electrode 98

3.3.2 Channel-flow electrode 105

$\begin{array}{ll}\text { 3.3.3 Wall-jet electrode } & 107\end{array}$

3.4 Non-stationary or transient electrode processes $\quad 110$

3.4.1 Introduction 110

3.4.2 Current step in the absence of mixing 112

3.4.3 Current step with mixing 115

3.4.4 Current scan 118

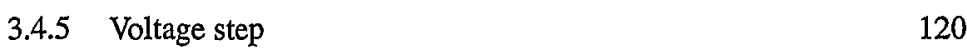

Exercises 122

References 124

4 Transport in membranes 126

4.1 Transport across neutral porous membranes 126

4.1.1 Quasi-steady diffusive transport between two closed compartments

4.1.2 Lag time in diffusion $\quad 129$

4.1.3 Tontophoretic enhancement 133

4.1.4 Lag time in electrodiffusion $\quad 136$

4.1.5 Circulation of compartments 139

4.1.6 Convective electrophoresis $\quad 140$

4.1.7 Liquid-junction potential 143

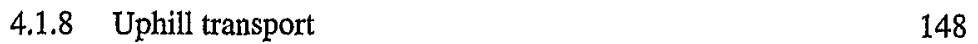


4.2 Donnan equilibrium in charged membranes 152

4.2.1 Ion-exchange membranes 152

4.2.2 Donnan equilibrium 152

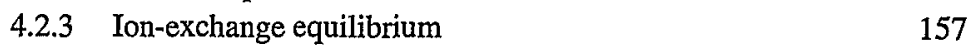

4.2.4 Electrical double layer at the membrane/ external solution interface 160

4.2.5 Influence of the membrane heterogeneities 163

4.3 Steady-state transport across ion-exchange membranes 167

4.3.1 Transport coefficients and their equilibrium values 167

4.3.2 The diffusion-conduction flux equation inside charged membranes $\quad 170$

4.3.3 Diffusion of a binary electrolyte $\quad 173$

4.3.4 Membrane permselectivity 182

4.3.5 Counterion interdiffusion through an ideally permselective membrane 191

4.3.6 Bi-ionic potential 195

4.3.7 Transport in multi-ionic solutions 196

4.3.8 Concentration polarization in ion-exchange
membrane systems

4.3.9 Influence of the diffusion boundary layers

4.4 Steady-state transport across charged porous membranes 205

4.4.1 The radial electrical double layer 205

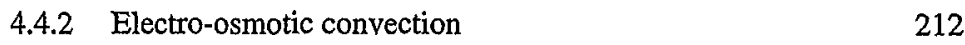

4.4.3 Transport mechanisms in charged porous membranes 217

4.4.4 Theoretical approaches for describing transport in porous membranes $\quad 219$

Exercises 221

References 229

\section{Transport through liquid membranes 232}

5.1 Distribution equilibria in liquid membrane systems 232

5.1.1 Liquid membranes 232

5.1.2 Equilibrium partitioning at the aqueous/organic solution interface

5.1.3 Finite-volume effects on the partitioning of a neutral solute

5.1.4 Finite-volume effects on the partitioning of an ionic solute

5.2 Ion transfer across a liquid membrane $\quad 239$

5.3 Carrier-mediated transport 243

5.3.1 Solute permeability in a supported liquid membrane 243

5.3.2 Free solute transport: the solubility-diffusion mechanism 244

5.3.3 The effect of the diffusion boundary layers on free solute transport 
5.3.4 Carrier-mediated solute transport: the facilitation factor

5.3.5 The effect of the diffusion boundary layers on carrier-mediated transport

5.3.6 Extraction of an acid

5.3.7 Additional comments on the modelling of carrier-mediated transport

5.4 Carrier-mediated coupled transport

5.4.1 Introduction

5.4.2 Competitive binding of two neutral solutes to a carrier

5.4.3 Sequential binding of two neutral solutes to a carrier

5.4.4 Polyelectrolyte extraction by carrier-mediated co-transport with an acid

5.4.5 Polyelectrolyte extraction by carrier-mediated countertransport with an acid

References 